\title{
Inverse Grain-Size Effect on Twinning in Nanocrystalline Ni
}

\author{
X. L. $\mathrm{Wu}^{1, *}$ and Y.T. $\mathrm{Zhu}^{2, \dagger}$ \\ ${ }^{1}$ State Key Laboratory of Nonlinear Mechanics, Institute of Mechanics, Chinese Academy of Sciences, Beijing 100190, China \\ ${ }^{2}$ Department of Materials Science and Engineering, North Carolina State University, Raleigh, North Carolina 27695-7907, USA
}

(Received 18 February 2008; revised manuscript received 25 May 2008; published 10 July 2008)

\begin{abstract}
A long-standing controversy exists between molecular dynamics simulations and experiments on the twinning propensity of nanocrystalline (NC) face-centered-cubic metals. For example, three-dimensional molecular dynamics simulations rarely observed twins in $\mathrm{NC} \mathrm{Ni}$, whereas experiments readily observed them. Here this discrepancy is resolved by experimental observation of an inverse grain-size effect on twinning. Specifically, decreasing the grain size first promotes twinning in $\mathrm{NC} \mathrm{Ni}$ and then hinders twinning due to the inverse grain-size effect. Interestingly, no inverse grain-size effect exists on stacking fault formation. These observations are explained by generalized planar fault energies and grain-size effect on partial emissions.
\end{abstract}

PACS numbers: $61.82 . \mathrm{Rx}$

To twin or not to twin? This is a long-standing controversy in the community of nanocrystalline (NC) metals for many years. Specifically, molecular dynamics (MD) simulations of three-dimensional (3D) grains rarely observed deformation twins in $\mathrm{NC} \mathrm{Ni}$ and $\mathrm{Cu}$ [1-3], whereas experiments showed that bulk 3D $\mathrm{NC} \mathrm{Ni}$ and $\mathrm{Cu}$ deformed readily by twinning [4-8]. This discrepancy between MD simulations and experiments raises a serious question: How well can MD simulations predict the deformation mechanisms in real NC materials?

Before further deliberating on the above question, let us first briefly delineate some significant 3D MD simulation results on NC face-centered-cubic (fcc) metals. Yamakov et al. [9] recently presented a deformation-mechanism map for 3D NC fcc metals, which shows that larger grains tend to deform by slip of perfect dislocations, whereas smaller grains tend to deform by slip of Shockley partials. But they did not clarify if those partial slips also produced twins. In a systematic study, Van Swygenhoven et al. [1,2] found that $\mathrm{NC} \mathrm{Cu}$ and Ni primarily deformed by single Shockley partials, which produced stacking faults, but rarely observed deformation twins. They attributed the lack of twinning to higher activation energy (or stress) for twin nucleation than for stacking fault nucleation on the general planar fault energy (GPFE) curves and to the high strain rates of MD simulations [1]. However, a recent MD simulation shows that the high strain rates should promote twinning [10,11].

Experimentally, stacking faults are observed in $\mathrm{NC} \mathrm{Cu}$ and $\mathrm{Ni}$ when their grains are smaller than certain critical sizes, which is consistent with the deformation-mechanism map [9]. However, twins are also readily observed in them [4-8,12], which contradicts the 3D MD simulations $[1,2,9]$. To explain this discrepancy on twinning between the 3D MD simulations and experiments, factors other than GPFE curves have been proposed, including nonequilibrium grain boundaries [12], stress concentrations [5,12,13], and favorable orientations of twinning partials [5,12].
However, it is not clear if any of these factors play a dominant role in determining the partial slips. Since the deformation twinning significantly affects the mechanical properties of NC materials, especially the ductility [14], it is of critical importance to solve this discrepancy if the MD simulations are to be used to guide the design of $\mathrm{NC}$ materials [10].

Here we present experimental evidence that the discrepancy on twinning is caused by a previously unknown inverse grain-size effect on twinning, in which the twinning propensity decreases with decreasing grain size. It contrasts with the reported normal grain-size effect of higher twinning propensity with decreasing grain size in $\mathrm{NC}$ fcc metals $[4,6,7,9]$. As shown later, the normal grainsize effect takes effect in larger $\mathrm{NC}$ grains, while the inverse grain-size effect takes effect in smaller NC grains.

An electrodeposited NC Ni foil with grains in the range of $10-75 \mathrm{~nm}$ and an average grain size of $\sim 25 \mathrm{~nm}$ was deformed at liquid nitrogen temperature under three deformation modes with increasing strain rate and strain: (1) quasistatic tension at a strain rate of $3 \times 10^{-3} \mathrm{~s}^{-1}$ to a strain of $5.5 \%$ and at a flow stress of $1.5 \mathrm{GPa},(2)$ rolling at a strain rate of $2 \times 10^{-2} \mathrm{~s}^{-1}$ to a strain of $9.8 \%$, and (3) split Hopkinson pressure bar (SHPB) test at a strain rate of $\sim 2.6 \times 10^{3} \mathrm{~s}^{-1}$ to a strain of $13.5 \%$ and a flow stress of $\sim 2 \mathrm{GPa}$. We examined about 130 grains for each sample using high-resolution electron microscopy (HREM) and found grains containing twins, stacking faults, and full dislocations as well as "clean" grains. Figure 1 shows typical HREM images of deformation twins and stacking faults. No grain growth was observed after these deformations. All samples showed a significant fraction (40\%$45 \%$ ) of clean grains in which no twins, stacking faults, or dislocations were observed. However, some of these clean grains may actually contain defects, but their orientation is such that HREM images do not reveal them [6].

Figure 2 shows the histograms of (a) grain-size distribution, (b) the number of grains containing stacking faults 

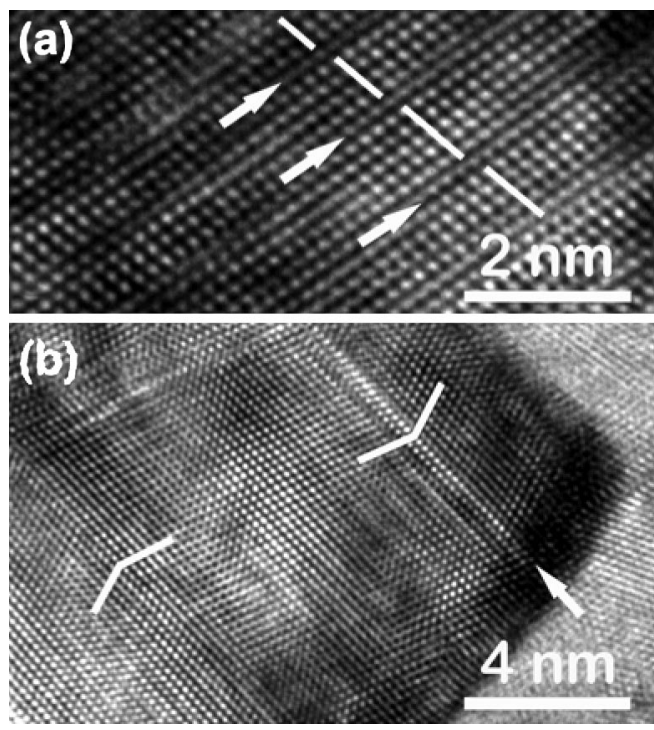

FIG. 1. Typical HREM images of (a) stacking faults and (b) deformation twins in $\mathrm{NC} \mathrm{Ni}$ after tension and the split Hopkinson pressure bar test at liquid nitrogen temperature.

and twins, and (c) fractions of grains containing stacking faults and twins in samples deformed under tension. Figure 2(b) shows that with decreasing grain size the number of grains containing twins (hereafter referred to as twinned grains) first increases and then decreases, while the number of grains containing stacking faults increases monotonically. The fraction of twinned grains is a better statistical indicator of twinning propensity. As shown in Fig. 2(c), the twinning propensity clearly drops with decreasing grain size for grains smaller than $34 \mathrm{~nm}$, which is a typical inverse grain-size effect by definition. In contrast, no inverse grain-size effect exists for stacking faults.

When the NC Ni samples were deformed at a higher strain rate by rolling, deformation twinning became more prevalent (see Fig. 3). Figure 3(b) shows the same trend of twinning propensity with grain size as that shown in Fig. 2(b). However, the largest grain that contains a stacking fault dropped from $38 \mathrm{~nm}$ in Fig. 2(b) to $26 \mathrm{~nm}$ in Fig. 3(b), indicating that higher strain rate and strain enabled smaller grains to deform by twinning. The effect of grain size on twinning propensity is demonstrated more clearly in Fig. 3(c). Disregarding the one data point at $63 \mathrm{~nm}$ because it has only one grain, we can clearly see that the fraction of twinned grains peaks at a grain size around $35 \mathrm{~nm}$, and an inverse grain-size effect is revealed in grains smaller than $35 \mathrm{~nm}$.

Figure 4 shows the histograms illustrating the effect of grain sizes on the deformation twins and stacking faults formed by deforming the $\mathrm{NC} \mathrm{Ni}$ at the highest strain rate $\left(\sim 2.6 \times 10^{3} \mathrm{~s}^{-1}\right)$ by SHPB tests. Figure 4(b) shows that the twinned grains are larger than $16 \mathrm{~nm}$, while grains containing stacking faults are smaller than $16 \mathrm{~nm}$. Figure 4(c) clearly shows an inverse grain-size effect in grains smaller than $50 \mathrm{~nm}$.

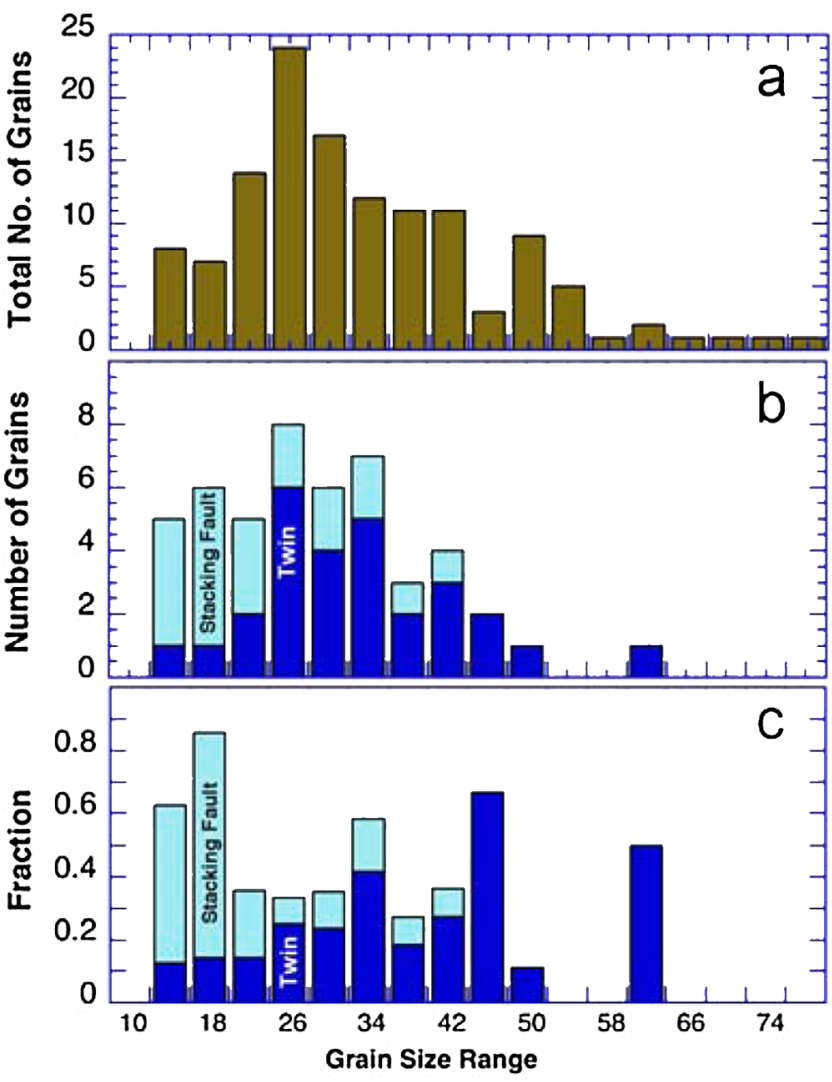

FIG. 2 (color online). Statistical grain-size effect on the formations of stacking faults and deformation twins in $\mathrm{NC} \mathrm{Ni}$ deformed under tension at liquid nitrogen temperature. (a) The size distribution of all grains examined under TEM. (b) The number distributions of grains containing stacking faults and twins. (c) The fraction distribution of grains containing stacking faults and twins. Each fraction was calculated as the ratio of the number of grains containing stacking faults and twins to the total number of grains in the corresponding size range.

Figure 5(a) shows that the average size of twinned grains is larger than $25 \mathrm{~nm}$, the average size of all grains in the sample, while the average size of grains that contain stacking faults is smaller than $25 \mathrm{~nm}$ in NC Ni samples deformed under all of the three modes. This indicates that deformation twins tend to form in large grains, while stacking faults tend to remain in smaller grains. In addition, with increasing strain rate and strain, the average size of twinned grains increases, which suggests that higher strain rate and strain make it easier for larger grains to deform by twinning. At the same time, Fig. 5(a) also shows that the maximum size of grains containing stacking faults drops dramatically with increasing strain rate and strain, which indicates that higher strain rate and strain also make it easier for smaller grains to convert stacking faults to twins. In other words, higher strain rate and strain enabled the twins to form more easily in both larger grains and smaller grains.

Figure 5 also indicates the existence of an optimum grain-size range for deformation twinning, which is con- 


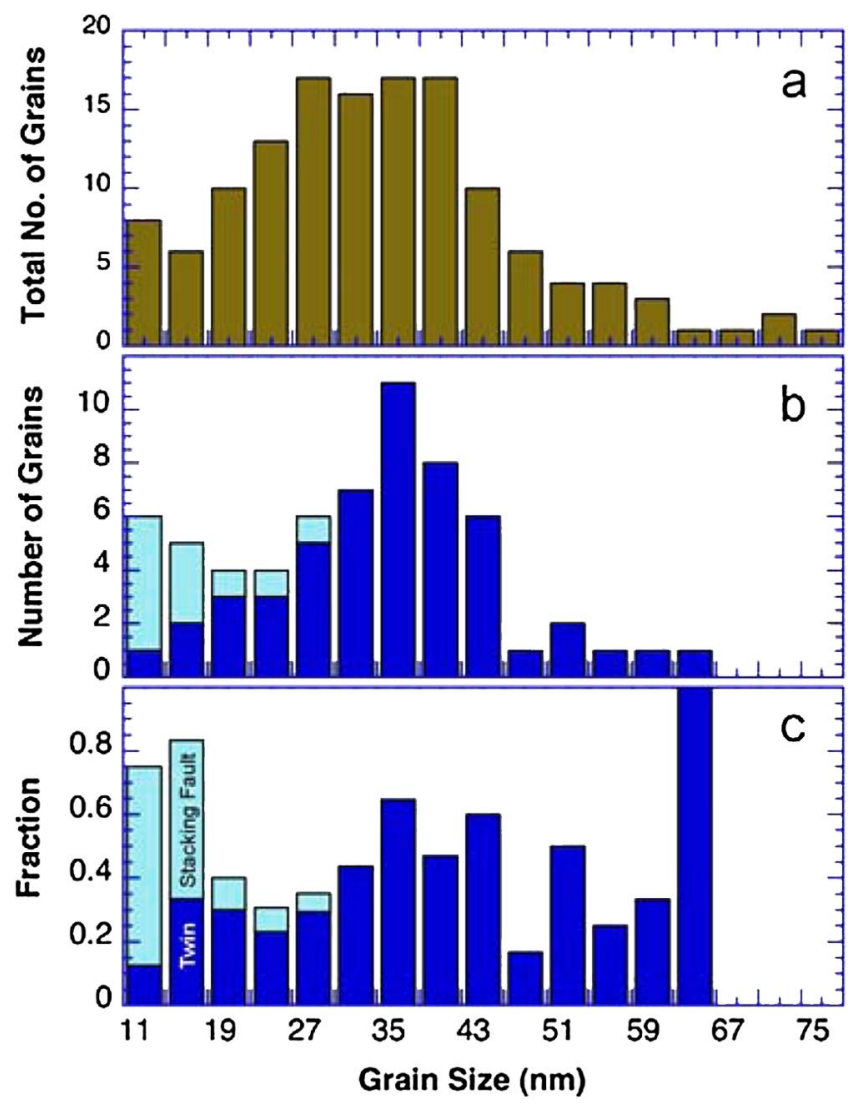

FIG. 3 (color online). Statistical grain-size effect on the formations of stacking faults and deformation twins in $\mathrm{NC} \mathrm{Ni}$ deformed by cryorolling.

sistent with a previous analytical model $[15,16]$. The precondition for the nucleation of a deformation twin is to first form a stacking fault. In other words, a stacking fault was first formed by a leading partial, and then a twinning partial converted the stacking fault to a twin nucleus. This suggests that leading partials were first activated in grains with a wide range of sizes to create stacking faults, but twinning partials become more difficult to nucleate and slip with decreasing grain size if the inverse grain-size effect is operating. Therefore, it takes higher applied stress to emit a twinning partial than an extended partial.

The higher stress requirement for activating twinning partials is consistent with the effect of GPFE energies on partial slips, which was recently predicted by MD simulations [3,17-19] and observed experimentally [5,6]. This was also attested by the fact that the fraction of twinned grains increased from $28 \%$ under tension to $38 \%$ under rolling to 44\% under SHPB [see Fig. 5(b)]. The flow stress under tension was $1.5 \mathrm{GPa}$, and this was increased to 2.0 GPa under SHPB [6,10]. Although the flow stress under rolling could not be measured, it should be between 1.5 and 2.0 GPa, because higher strain rate usually leads to higher flow stress. This observation is somewhat different from that in a recent study using laser-driven compression at an

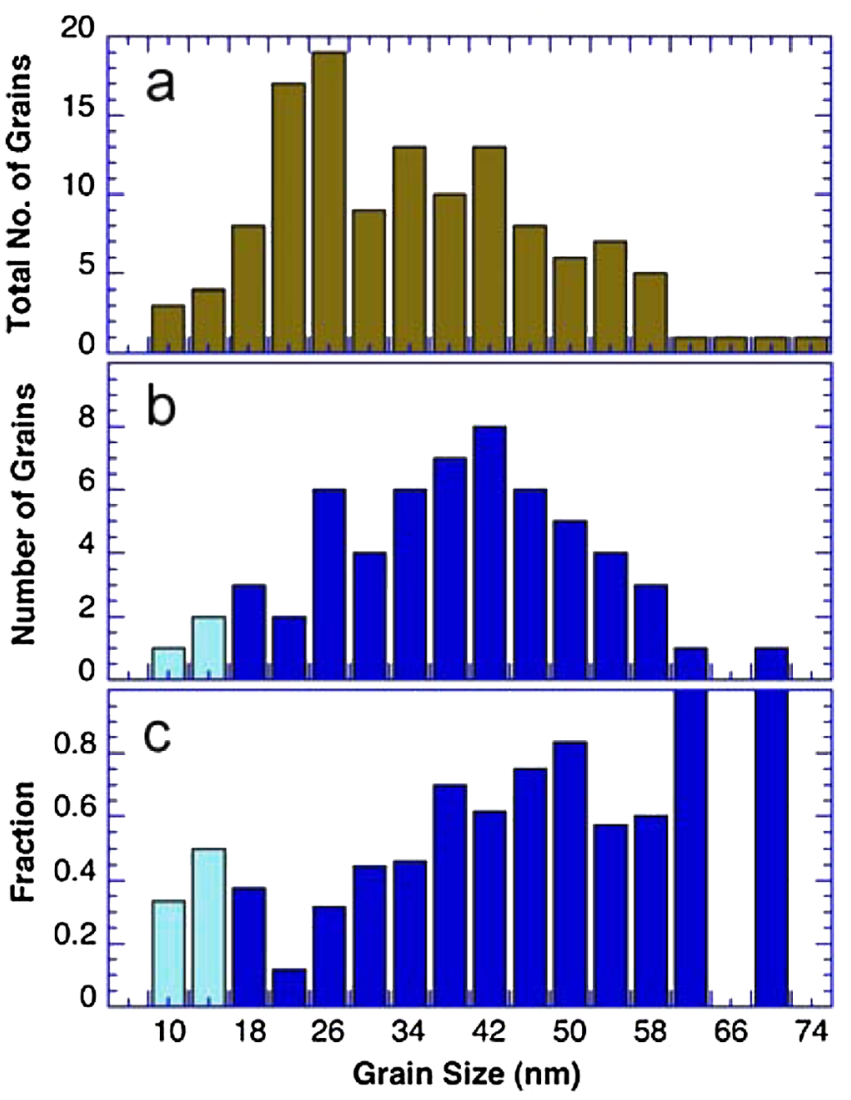

FIG. 4 (color online). Statistical grain-size effect on the formations of stacking faults and deformation twins in $\mathrm{NC} \mathrm{Ni}$ deformed by SHPB at liquid nitrogen temperature.

extremely high strain rate of $>10^{7} \mathrm{~s}^{-1}$ [20] but consistent with a recent $\mathrm{MD}$ simulation on the strain rate effect $[10,11]$.

The inverse grain-size effect on twinning can be explained by the combination of the GPFE energies and the grain-size effect on emission of partials. It has been reported that higher stresses are required to emit partials in smaller grains $[9,15,21]$. Combined with the requirement of higher stress to emit twinning partials than leading partials, one can envision that below a certain critical grain size the stress required to emit a twinning partial becomes higher than the applied stress, which stops twinning but still allows the emission of the leading partials to create extended stacking faults. Because of the variation in grain orientations and local stress states, this leads to a statistical decrease in twin density with decreasing grain size, i.e., the inverse grain-size effect on twinning.

Note that the electrodeposited NC Ni film might have a texture. If smaller grains had been unfavorably oriented for twinning, it would explain the observed inverse grain-size effort. However, this is clearly not the case in this study because the tension deformed the sample along a direction parallel to the film surface, SHPB deformed the sample perpendicular to the film surface, while rolling deformed the sample in a more complex manner. Since the inverse 


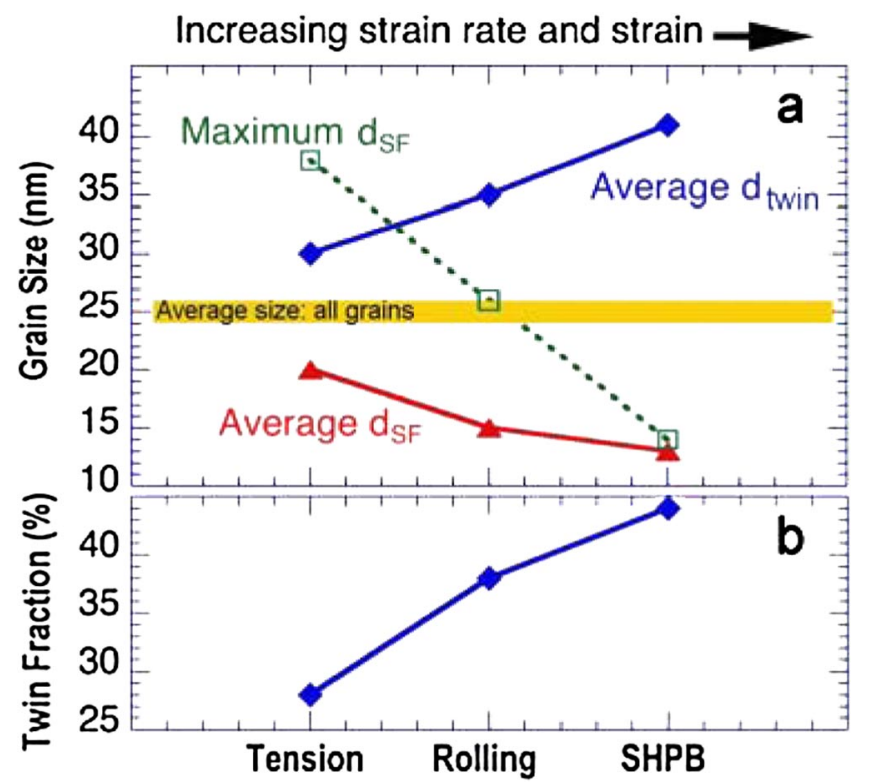

FIG. 5 (color online). (a) The average sizes of grains containing deformation twins and stacking faults as well as the maximum size of grains containing stacking faults; (b) fraction of twinned grains in NC Ni deformed at liquid nitrogen temperature under tension, rolling, and SHPB. Tension: strain rate $=3 \times$ $10^{-3} \mathrm{~s}^{-1}, \quad$ strain $=5.5 \%, \quad$ flow stress $=1.5 \mathrm{GPa} ; \quad$ rolling: strain rate $=2 \times 10^{-2} \mathrm{~s}^{-1}$, strain $=9.8 \%$; SHPB: strain rate $=$ $\sim 2.6 \times 10^{3} \mathrm{~s}^{-1}$, strain $=13.5 \%$, flow stress $=\sim 2 \mathrm{GPa}$.

grain-size effect was consistently observed in all 3 cases with different deformation directions, it could not have been caused by the variation of grain orientation with grain size.

The inverse grain-size effect observed here has a profound impact on how to compare MD simulations with experiments. In many MD simulations [1,13,22], the grain sizes are smaller than those in typical samples used in experiments. As a result, the MD simulation may predict more stacking faults and fewer twins than experimental observations. This is the reason for the current controversy between 3D MD simulations and experiments.

In summary, statistical data in this study clearly revealed an inverse grain-size effect on twinning in NC Ni below a certain critical grain size. However, no inverse grain-size effect was observed for the slips of leading partials, which produce stacking faults. There is an optimal grain-size range in which twinning is favored. The inverse grainsize effect has not been observed before either by MD simulations or by experiments. The results reported here suggest that both GPFE curves and grain size affect the slip of partials. The grain-size effect is primarily responsible for the reported controversies among 3D MD simulations and experimental observations. These observations should help with future design of NC materials using computer simulations.

X. L. W. was supported by NSFC Grants No. 50471086, No. 50571110, and No. 10721202, CAS Grant No. KJCX2-YW-M04, and MOST Grant No. 2004CB619305. Y.T.Z. was supported by the U.S. DOE IPP Program Office.

*xlwu@imech.ac.cn

†ytzhu@ncsu.edu

[1] H. Van Swygenhoven, P. M. Derlet, and A. G. Froseth, Nat. Mater. 3, 399 (2004).

[2] A. G. Froseth, P. M. Derlet, and H. Van Swygenhoven, Adv. Eng. Mater. 7, 16 (2005).

[3] H. Van Swygenhoven, P. M. Derlet, and A. Hasnaoui, Phys. Rev. B 66, 024101 (2002).

[4] X. L. Wu, Y. T. Zhu, M. W. Chen, and E. Ma, Scr. Mater. 54, 1685 (2006).

[5] X. L. Wu, Y.T. Zhu, and E. Ma, Appl. Phys. Lett. 88, 121905 (2006).

[6] X. L. Wu, Y. Qi, and Y.T. Zhu, Appl. Phys. Lett. 90, 221911 (2007).

[7] X.Z. Liao, Y.H. Zhao, S. G. Srinivasan, Y. T. Zhu, R. Z. Valiev, and D. V. Gunderov, Appl. Phys. Lett. 84, 592 (2004).

[8] X.Z. Liao, Y.H. Zhao, Y. T. Zhu, R.Z. Valiev, and D. V. Gunderov, J. Appl. Phys. 96, 636 (2004).

[9] V. Yamakov, D. Wolf, S. R. Phillpot, A. K. Mukherjee, and H. Gleiter, Nat. Mater. 3, 43 (2004).

[10] D. H. Warner, W. A. Curtin, and S. Qu, Nat. Mater. 6, 876 (2007).

[11] V.I. Yamakov and E. H. Glaessgen, Nat. Mater. 6, 795 (2007).

[12] X. L. Wu and Y. T. Zhu, Appl. Phys. Lett. 89, 031922 (2006).

[13] J. Wang and H. Huang, Appl. Phys. Lett. 85, 5983 (2004).

[14] Y.H. Zhao, Y. T. Zhu, X.Z. Liao, Z. Horita, and T. G. Langdon, Appl. Phys. Lett. 89, 121906 (2006).

[15] Y. T. Zhu, X.Z. Liao, S. G. Srinivasan, Y. H. Zhao, M. I. Baskes, F. Zhou, and E. J. Lavernia, Appl. Phys. Lett. 85, 5049 (2004).

[16] Y. T. Zhu, X. Z. Liao, S. G. Srinivasan, and E. J. Lavernia, J. Appl. Phys. 98, 034319 (2005).

[17] R. J. Asaro and S. Suresh, Acta Mater. 53, 3369 (2005).

[18] J. R. Rice, J. Mech. Phys. Solids 40, 239 (1992).

[19] E. B. Tadmor and S. Hai, J. Mech. Phys. Solids 51, 765 (2003).

[20] Y.M. Wang, E. M. Bringa, and J.M. McNaney et al., Appl. Phys. Lett. 88, 061917 (2006).

[21] S. Cheng, J. A. Spencer, and W. W. Milligan, Acta Mater. 51, 4505 (2003).

[22] H. Van Swygenhoven, P. M. Derlet, and A. G. Froseth, Acta Mater. 54, 1975 (2006). 